\title{
Evaluación del impacto comercial de las materias primas: una mirada al Acuerdo de la Asociación Regional (AAR) entre UE- MERCOSUR
}

\author{
Evaluation of the commercial impact of raw materials: a look at the Regional \\ partnership agreement between the European Union (EU) and the MERCOSUR
}

\author{
Víctor Ramón Enciso Cano ${ }^{1 *}$ \\ ${ }^{1}$ Facultad de Ciencias Agrarias, Universidad Nacional de Asunción, Campus Universitario, Ruta Mcal. Estigarribia, \\ Km 10,5, San Lorenzo, Paraguay. \\ *Autor para correspondencia (venciso@agr.una.py) \\ Recibido: 20/06/2016; Aceptado: 07/09/2016. \\ 10.18004/investig.agrar.2016.diciembre.61-69
}

\section{RESUMEN}

La Unión Europea (UE) y el Mercado Común del Sur (MERCOSUR) se hallan negociando la firma de un Acuerdo de Asociación Regional (AAR) desde mediados de los noventa, y continúan a la fecha. Los posibles efectos del AAR en el MERCOSUR han sido ampliamente estudiados. La gran mayoría de ellos coinciden en que el acuerdo beneficiará a las partes, y en el caso específico del MERCOSUR fortalecerá sus ventajas comparativas. El punto argumentado en el trabajo es demostrar que la implementación del Acuerdo condenará a los países del MERCOSUR a ser eternos exportadores de materias primas agrícolas y sus primeras transformaciones industriales, que es donde radican las ventajas comparativas del bloque. Entonces el Acuerdo dejará al MERCOSUR, sin o con escasa oportunidad para desarrollar manufacturas de mayor valor agregado. El trabajo concluye que el Acuerdo fortalecerá la exportación de bienes en los cuales el MERCOSUR tiene ventjas comparativas, que es lo mismo que decir será un eterno exportador de materias primas agrícolas y sus primeras manufacturas.

Palabras clave: MERCOSUR, UE, acuerdos comerciales, material prima, impactos comerciales.

\section{INTRODUCCIÓN}

La Unión Europea (UE) y el Mercado Común del Sur (MERCOSUR) se hallan negociando la firma de un Acuerdo de Asociación Regional (AAR) desde mediados

\begin{abstract}
The European Union (EU) and the Southern Common Market (MERCOSUR for its Spanish acronym) have been negotiating since the mid-nineties, to no avail, the signature of a bi-Regional partnership Agreement (AAR). The possible effects of the AAR on the MERCOSUR have been widely studied. Most of the studies agree that the agreement would be beneficial for both parties, and that in the specific case of Mercosur, the agreement would strengthen its comparative advantages.

The aim of this work is, however, to show that the implementation of the agreement would condemn the Mercosur countries to remain forever as exporters of agriculture raw material and manufactured goods with low added value, which is indeed, the block's main competitive advantages. In this context, the agreement will leave the MERCOSUR countries with scarce or no opportunity to develop industries with higher added value. This paper concludes that the Agreement will favor the export of products in which the MERCOSUR has already had competitive, which implies that MERCOSUR will remain as an exporter of agricultural commodities and producer of low added value manufactures
\end{abstract}

Key words: MERCOSUR, UE, commercial agreements, raw material, trade impact.

de los noventa. El Acuerdo que abarca el diálogo político, la cooperación y el establecimiento de una zona de libre comercio, se incluye entre los denominados acuerdos de 
cuarta generación. Estos acuerdos más amplios que los tradicionales buscan formar una asociación política, económica, financiera, social y cultural, no solamente comercial. En setiembre de 2004, luego del intercambio de propuestas de acceso a mercado, las negociaciones fueron suspendidas. Años más tarde, en el 2010, fueron retomadas y a la fecha continúan sin haber llegado a un acuerdo. Recientemente, en Mayo del 2016 las partes intercambiaron propuestas de acceso a mercado.

Los posibles efectos del AAR en el MERCOSUR han sido ampliamente estudiados aplicando diversas metodologías desde los complejos modelos de equilibrio general hasta el uso de indicadores de comercio. Los estudios concluyen que el acuerdo será beneficioso para las partes, y que propiciará un intercambio comercial de acuerdo a las ventajas comparativas de los bloques. En otros términos, la UE exportará al MERCOSUR manufacturas industriales e importará bienes agrícolas y sus primeras transformaciones industriales. El documento consta, además de esta introducción, de un capítulo a modo de marco teórico sobre tipos de integración y metodologías de evaluación de impactos en acuerdos comerciales. Le sigue la metodología utilizada y un capítulo de resultado y discusiones.Finalmente se presentan las conclusiones y se dan algunas recomendaciones prácticas derivadas del análisis.

\section{DESARROLLO}

\section{La integración comercial}

La integración se puede definir como una unión o instancia en la cual los países o las economías se unen con el objetivo de acelerar su tasa de expansión global y racionalizar su crecimiento. Maesso (2011) indica que los primeros antecedentes de la integración se pueden encontrar en la Gran Bretaña del siglo XVIII, y en la Alemania e Italia del siglo XIX. Sin embargo, el fenómeno integracionista toma impulso tras la Segunda Guerra Mundial siendo la Comunidad Económica Europea (CEE) el ejemplo más importante. Otras iniciativas tuvieron menos éxito por diversos motivos, según Bhagwati (1992) principalmente debido a la reticencia de Estados Unidos en aplicar el artículo XXIV del GATT. Este artículo básicamente permite los acuerdos regionales siempre que una parte sustancial del comercio sea liberado, pero sin especificar la magnitud de tal liberación.
La segunda ola de regionalismo, que se inició en los 80s, tuvo mayor éxito debido en gran parte a iniciativas lideradas por los Estados Unidos, que a partir de esta década ya hizó uso del artículo XXIV del GATT (Bhagwati 1992). Desde entonces han surgido diversos acuerdos importantes entre los cuales se puede identificar el Tratado de Libre Comercio de América del Norte, la profundización de la Unión Europea, la conformación de bloques económicos en Asia como la Asociación de Naciones del Sudeste Asiático (ASEAN) o el Mercado Común del Sur (MERCOSUR). En el presente milenio hay que destacar, el Acuerdo Transpacífico de Cooperación Económica, más conocido por sus siglas en inglés, TPP, que involucra a economías del Pacifico entre ellas, EEUU, Canadá, México, Perú, Malasia, Japón, Australia y Vietnam, y las negociaciones entre la UE y los Estados Unidos conocido como Tratado Transatlántico de Comercio e Inversiones (TTIP). A la fecha, la Base de Datos de la Organización Mundial de Comercio (OMC) tiene registrado 277 Acuerdos Comerciales en implementación.

Una de las justificaciones de la integración económica es la ampliación del mercado. Un mercado pequeño no es atractivo para las inversiones dado que el retorno a la inversión no sería rentable con la demanda de una economía pequeña. Por el contrario, un mercado ampliado incrementa la demanda, permite obtener economías de escala y de especialización, al tiempo que aumenta y fortalece el poder de negociación del grupo en relación a la capacidad individual de sus integrantes. Además, la integración fomenta a medio plazo la competencia, la disminución de los costos y la movilidad de los factores de producción promoviendo la mejora de la eficiencia. De igual manera en el largo plazo se observan efectos sobre las variables de crecimiento, a saber cambios en la estructura de producción, difusión de tecnología y facilidad para acceder al mercado de capitales.

La integración se clasifica tradicionalmente en cinco categorías en función del nivel de profundización: (i) zona de libre comercio, (ii) unión aduanera, (iii) mercado común, (iv) unión económica, (v) unión total (Balassa 1965). La forma más elemental y más utilizada la constituyen el área o zona de libre comercio que se forma cuando las partes firmantes del acuerdo deciden eliminar las restricciones al comercio entre ellas, pero manteniendo su independencia en cuanto a sus aranceles con terceros países. Un ejemplo de este tipo de acuerdo es el Tratado de Libre Comercio de América del Norte entre México, Estados Unidos y Canadá que está en vigencia desde el 1 
de Enero de 1994. La independencia de cada país en su política comercial se evidencia en el hecho de que México y Canadá tienen un Tratado de Libre Comercio con la Unión Europea desde el 2000 y el 2015 respectivamente, mientras que Estados Unidos está actualmente en etapa de negociación con la UE para el establecimiento de un acuerdo similar.

Por otro lado, Solares (2010) argumenta que los Sistemas de Preferencias Arancelarias constituyen la forma más elemental de integración. Implica que las partes se conceden mutuamente rebajas arancelarias, pero no extensibles a terceros. La vigencia del GATT en 1947 y con ellas la cláusula de Nación Más Favorecida imposibilitó nuevos acuerdos de este tipo, pero no eliminó los vigentes. Si bien las preferencias bilaterales han desaparecido, han surgido otras de carácter unilateral denominadas los Sistemas Generalizados de Preferencias (SGP), que son aplicados por países desarrollados (EEUU, Japón y la UE) en favor de las exportaciones de países en desarrollo sin exigir reciprocidad.

Cuando los integrantes de un Área de Libre Comercio deciden establecer un Arancel Externo Común (AEC) se llega a una Unión Aduanera. Se tiene un Mercado Común cuando además de la libre circulación de bienes y el establecimiento de un AEC se acuerda la libre circulación de los factores de producción, personas, capitales y servicios. La Unión Europea en su proceso de integración durante los noventa puede ser considerado como un ejemplo de Unión Aduanera. Cuando los países integrados en un Mercado Común deciden avanzar hacia la armonización de políticas fiscales y monetarias se llega a lo que se denomina Unión Económica. La actual UE surgida del Tratado de Maastricht es lo más cercano a una Unión Económica que se conozca. Finalmente y más bien como un objetivo ideal se incluye la Integración Política que implica la unificación de todas las políticas, planes y legislaciones económicas (Solares 2010).

\section{Los efectos de los acuerdos comerciales}

El análisis económico sobre los efectos de los acuerdos comerciales tiene su inicio en el trabajo de Viner (1950), sobre teoría de las uniones aduaneras. Hasta ese momento los acuerdos comerciales se analizaban con las mismas metodologías aplicadas a la defensa del libre comercio, a saber las ventajas comparativas de Ricardo, o la de dotación de factores de Heckscher-Ohlin, entre las más tradicionales. El trabajo de Jacob Viner identificó dos efectos sobre la producción y los flujos comerciales como consecuencia de la integración. Por un lado, el efecto positivo de la creación de comercio, y por el otro, el efecto negativo de desviación de comercio. Estos efectos son conocidos como estáticos o de corto plazo, para diferenciarlos de los efectos dinámicos o de largo plazo.

La creación de comercio tiene lugar cuando una de las partes del acuerdo deja de producir un producto para sustituirlo por otro que es importado del nuevo socio comercial. Se considera que con esto se mejora la asignación de recursos y el bienestar general, al evidenciar las ventajas de la especialización y el comercio de los países que se integran. Es un efecto positivo del acuerdo comercial.

Por su lado en el desvío del comercio una de las partes reemplaza la importación de un producto desde un país que además de no formar parte del acuerdo, es más eficiente en la producción del mismo, por otro producto bien similar proveniente de uno de los nuevos socios comerciales. Este nuevo proveedor tiene ventajas sobre el anterior no debido a una mayor eficiencia, sino a las concesiones preferenciales del acuerdo. Como resultados, el país más eficiente es dejado de lado por otro menos eficiente. Es un efecto negativo del acuerdo comercial.

Posterior al trabajo de Viner, Meade y Lipsey (citado Maesso 2011) sustituyeron el supuesto de Vinner de que los bienes son consumidos en proporciones fijas, y asumieron que el consumo variaba en función a los precios relativos. En otros términos, el comercio se expandía, decían Meade y Lipsey, debido a las mayores importaciones que respondían a su vez al aumento del consumo, y éste era una consecuencia de la reducción del precio propiciada por la integración. Con esto añadieron un tercer efecto, la expansión del comercio. Es un efecto positivo del acuerdo comercial.

El efecto final de la integración puede ser positivo o negativo dependiendo de la importancia de los efectos positivos (incremento del comercio y expansión del comercio) y de los efectos negativos (desviación del comercio). Así por ejemplo, la creación de la Unión Europea ha supuesto un importante efecto de desviación de comercio en el sector agrario que ha sido más que compensado por un efecto positivo de creación de comercio en otros sectores.

Los principales efectos dinámicos se observan en las economías de escala, en la competencia y por extensión en el crecimiento económico. Según Trejos (2009), "la 
desgravación genera efectos dinámicos asociados al potencial aumento de la competencia, el mayor acceso a mercados internacionales, los flujos de inversión extranjera directa (IED) y la variación en los canales de transferencia tecnológica, entre otros factores". Maesso (2011) agrega a todo esto los beneficios provenientes del aprovechamiento de las ventajas comparativas o sea de la diferencia en la dotación relativa de factores de producción o en el nivel de desarrollo tecnológico.

\section{Metodologías de evaluación de los acuerdos comerciales}

Existen diversas metodologías para evaluar el impacto de las políticas comerciales per se o de aquellas que surgen como consecuencia de acuerdos comerciales firmados entre las partes involucradas. CEI (2003) las agrupa en tres niveles en función al grado de agregación de los productos. En el primer nivel, el más agregado de todos, están los modelos cuantitativos a saber equilibrio general computable. En un segundo nivel de desagregación están los análisis realizados mediante el uso de indicadores de comercio exterior y barreras comerciales. En el nivel más desagregado están los estudios sectoriales.

El uso de modelos de equilibrio parcial puede estar en el primer como en el segundo nivel dependiendo del grado de desagregación. Por ejemplo, un estudio del impacto que involucre al cultivo de trigo en forma específica, correspondería al segundo nivel, mientras que si se focaliza en los cereales como conjunto correspondería a un nivel de mayor agregación. Específicamente las evaluaciones utilizando modelización, según Piermartini y Teh (2005), pueden clasificarse de acuerdo a dos criterios. El primero tiene en cuenta el periodo de tiempo en que realiza la evaluación, pudiendo entonces ser evaluaciones ex ante o evaluaciones ex post. El segundo criterio sostiene que el análisis puede ser estático o dinámico, en este caso se tiene en cuenta la forma de analizar el efecto del cambio en la situación de equilibrio inicial, previo a la entrada en vigencia del Acuerdo. UNCTAD (2012) realiza la siguiente clasificación (i) estadísticas descriptivas y modelización; (ii) estimaciones econométricas y simulaciones; (iii) evaluaciones ex ante y ex post; (iv) equilibrio parcial y equilibrio general.

\section{Hipotesis y metodologia}

Este trabajo busca demostrar que el Acuerdo Comercial en negociación entre la Union Europea y el Mercado Común del Sur (MERCOSUR) consolidará la especialización de este último en la producción y exportacion de bienes primarios agrícolas y sus primeras manufacturas mas comúnmente denominados agroalimentos. El argumento a favor es que el MERCOSUR está compuesto por países especializados y presenta ventajas comparativas en la producción de agroalimentos, de modo que el acuerdo reforzará dicha situación.

Dentro de esta hipotésis de trabajo, esta investigación apunta a demostrar que los resultados de los diversos estudios de impacto del acuerdo, sean los hechos con modelos de equilibrio general computable, o de equilibrio parcial, o indicadores de comercio, todos concluyen que el MERCOSUR obtendrá ventajas en la exportación de bienes agroalimentarios, agrícolas primarios o sus primeras transformaciones industriales (aceites, preparados alimenticios, harinas, etc).

A ese efecto se aplica una metodología cualitativa de análisis documental. Más específicamente se aplicó una estrategia cualitativa de recolección de información documental de tipo secundaria, siguiendo a Denzin y Lincoln (2000) y Folgueiras y Martines (2009). Los documentos a los que se recurre son los estudios de impacto del acuerdo comercial. Estos estudios fueron realizados utilizando diferentes metodologías, tales como modelos de equilibrio general computable, modelos de equilibrio parcial e indicadores de comercio las que son descriptas infra. Si bien generalmente este tipo de metodologia se utiliza para "desarrollar teorías" (Denzin y Lincoln 2000), en la presente investigación se utiliza para demostrar la hipótesis antes descripta. Estos productos son aquellos que formando parte de la actual oferta exportadora de los países incrementarían su exportaciones a la UE en el escenario con Acuerdo.

Una vez descripto el marco de los acuerdos comerciales y sus metodologías, expuesta la hipótesis, se establece como objetivo de este trabajo demostrar que la implementación del Acuerdo Comercial entre la UE y el MERCOSUR favorecerá o incentivará a los países del MERCOSUR a ser eternos exportadores de materias primas agrícolas y sus primeras transformaciones industriales, sin o con escasa oportunidad para desarrollar manufacturas de mayor valor agregado. Estos, a los efectos de este estudio, son aquellos bienes incluidos en los capítulos 1 al 24 del Sistema Armonizado de Designación y Codificación de Mercancias. De modo que cuando un estudio concluye que la implementación del Acuerdo incrementará las exportaciones de un producto 
incluido en esos capítulos, entonces se cumple con la hipótesis propuesta en la presente investigación.

\section{Estudios con modelos de equilibrio general computable}

Monteagudo y Watanuki (2003) analizaron el impacto potencial en los países del MERCOSUR, y con más detalles en Argentina y Brasil, de los Acuerdos ALCA y UE-MERCOSUR, tanto de forma separada como conjunta. Utilizaron como año base datos de 1997. Sus resultados indican que el Acuerdo con la UE representa para el MERCOSUR un crecimiento de las exportaciones del 7,91\%. Específicamente, en Argentina crecerán el $7,12 \%$ y en Brasil el 8,31\%. Más del $90 \%$ del incremento en las exportaciones del MERCOSUR están formados por productos relacionados con la agricultura (materia prima y productos agrícolas procesados). La implementación del Acuerdo ocasionaria que las exportaciones de los productos cárnicos crezcan más de 30 puntos porcentuales por encima del promedio en Argentina, y 20 puntos en el Brasil. En este último país también resultaron importantes los cambios en las exportaciones de granos que crecieron 40 puntos porcentuales sobre el promedio.

Drogue y Ramos (2005) focalizaron su estudio en el impacto que tendría en la economía comunitaria los contingentes arancelarios que formaban parte de la oferta de la UE de setiembre 2004 (Carne bovina, aviar, y porcina, trigo, maíz y lácteos). En términos comerciales, los resultados indicaron que las exportaciones de trigo desde el MERCOSUR a la UE disminuirían mientras que las carnes bovinas y otras carnes incrementarían su valor de exportación, concentrándose principalmente en la UE en detrimento de otros destinos.

Laborde y Ramos (2007) examinaron los flujos comerciales y sus efectos en el bienestar con y sin acuerdo comercial. Los resultados señalaron que la reducción de aranceles por parte de la UE y sin acuerdo en Doha, incrementaria las exportaciones totales de los países del MERCOSUR en al menos 2,5\% en Argentina, $7,4 \%$ en Brasil y 5,2\% en Paraguay y Uruguay. En el caso de Argentina, el comercio bilateral con la UE se incrementaría en lácteos $(27 \%)$ y carnes $(10 \%)$, vehículos automotores $(6 \%)$ y textiles (3\%). Brasil también aumentaría sus exportaciones a la UE especialmente en productos agroalimentarios (19\%), a saber lácteos, carnes, azúcar y arroz. Paraguay y Uruguay elevarían sus ventas a la UE en cereales, trigo, carnes, arroz y azúcar.
Kirkpatrick y George (2009) realizaron un estudio del impacto económico, social y ambiental de la implementación del acuerdo, tanto en la Unión Europea como en el MERCOSUR. Sus resultados indicaron que el valor de las exportaciones del MERCOSUR a la UE se incrementaria el 26\%, en relación al año base, en un escenario de liberalización total. Las exportaciones de alimentos procesados representaba el principal bien de exportación y donde se observaba el mayor incremento (210\%). La mayor participación en el aumento de las ventas al mercado comunitario correspondió al Brasil (75\%), seguido de Argentina (13\%), Paraguay (4\%) y Uruguay (3\%). Igualmente a nivel de cada país, los productos comprendidos en el sector de alimentos procesados fueron los que mayor aumento tuvieron en el valor de exportación. Las exportaciones paraguayas fueron las que mayor incremento tuvieron (42\%). En los demás países del MERCOSUR el aumento relativo de las exportaciones fueron también importantes: Brasil (38\%), Uruguay (27\%) y Argentina (14\%). Con la excepción de este último, en el caso de los demás países se pudo observar un incremento en las exportaciones de alimentos procesados y una disminución en todas las exportaciones de materia prima agrícolas debido a que éstas se utilizaron como insumos para los productos procesados.

Boyer y Schuschny (2010) modelaron el impacto del Acuerdo en un escenario de liberalización total y en otro en el cual se excluyeron los productos sensibles. Estos productos fueron para el MERCOSUR minerales, textiles, productos de cuero, maquinarias y equipos electrónicos, y para la UE arroz, carne y sus productos, lácteos, bebidas y tabaco. En el primer escenario las exportaciones del MERCOSUR a la UE crecen el 20\%. La mayor contribución al incremento provino de las manufacturas de origen agropecuario que incluyó a productos agrícolas procesados (carne, arroz, azúcar y productos alimenticios). Estos fueron los de mayor crecimiento en todos los países excepto en Uruguay donde los productos agrícolas sin procesar fueron los de mayor crecimiento. En el segundo escenario las exportaciones crecerían un $1,9 \%$, con los productos agrícolas (vegetales, frutas, nueces, cereales entre otros) incrementándose el 2,9\% y las manufacturas de origen agropeucario el $12,5 \%$. Los demás sectores mantenían sus valores negativos aunque en menor magnitud. Brasil y Paraguay tuvieron en las manufacturas de origen agropecuario su mayor crecimiento, mientras que Argentina y Uruguay en productos agrícolas. 
Burrel et al. (2011) simularon el impacto del Acuerdo utilizando cinco escenarios: I) oferta de la UE de septiembre 2004; II) oferta del MERCOSUR de 2006; III) finalización del acuerdo Doha en base a modalidades presentadas al comité de agricultura de la OMC en diciembre 2008; IV) acuerdo Doha + oferta UE adaptada con menores contingentes arancelarios al MERCOSUR; V) acuerdo Doha + oferta MERCOSUR. El estudio utilizó un modelo de equilibrio general (GLOBAL) para estimar los efectos en el conjunto de la economía, y un modelo de equilibrio parcial (CAPRI) para estimar los efectos en el sector agrario. Estos últimos se comentan junto con otros estudios de equilibrio parcial.

Los resultados de los escenarios I (oferta de la UE de setiembre 2004) y II (pedido del MERCOSUR del 2006), señalaban que el acuerdo incrementaría el valor de las importaciones europeas desde el MERCOSUR en 3,5\% y $4,2 \%$ respectivamente. Los bienes agroalimentarios, mayormente commodities con poco valor agregado, contribuyeron con el $67 \%$ y $73 \%$ del incremento en cada uno de los dos escenarios respectivamente. En el primer escenario los bienes de mayor contribución fueron: aceites y grasas (27\%), carnes bovina, caprina, ovina y caballar (8\%), alimentos, bebidas y tabaco (7\%), vegetales y frutas $(6 \%)$, carne porcina, aviar y otras $(5 \%)$, otros cultivos (4\%). En el segundo escenario los aportes principales provinieron de aceites y grasas $(23 \%)$, carnes bovina, caprina, ovina y caballar $(16 \%)$, carne porcina, aviar y otras (8\%), alimentos, bebidas y tabaco (4\%), vegetales y frutas (5\%). En relación a los contingentes arancelarios, en ambos escenarios las cuotas de arroz, sorgo, maíz, carnes y azúcar fueron completadas. En el segundo, el trigo se cubrió solamente un cuarto de su cuota, mientras que los productos lácteos (leche en polvo, mantequilla y queso) presentaron un porcentaje de llenado muy bajo.

En el escenario III, (acuerdo en Doha en base a modalidades presentadas al comité de agricultura de la OMC en diciembre de 2008), los resultados señalaron que las importaciones europeas desde el MERCOSUR crecerían en $6,3 \%$, de la cual el $80 \%$ sería debido a los productos agroalimentarios, en especial a la carne bovina con un aporte de 65 puntos porcentuales al total de agroalimentos. Otros productos de importancia fueron carne porcina, aviar y otras, vegetales y frutas y azúcar. Las importaciones europeas de oleaginosas y aceites vegetales disminuyeron en este escenario. En el cuarto escenario, (escenario III más la propuesta de la UE con cierta reducción en los contingentes arancelarios al
MERCOSUR), el incremento en el valor total importado desde el MERCOSUR fue del 8,5\% con un aporte de agroalimentos del $88 \%$. La tendencia se mantuvo en el último escenario, (escenario III más propuesta del escenario II), en el cual las importaciones desde el MERCOSUR crecerían el 9,23\% en relación al año base. En estos tres escenarios los contingentes arancelarios fueron totalmente utilizados con la excepción de lácteos y trigo.

\section{Estudios con modelos de equilibrio parcial}

Weissleder et al. (2008) evaluaron el acuerdo, utilizando CAPRI, que es un modelo de equilibrio parcial para el sector agrícola, desarrollado para evaluación de impacto de la Política Agrícola Común preferentemente. Se utilizaron dos líneas de bases. Una de ellas incluía la producción agrícola normal del MERCOSUR y en la otra se simulaba un fuerte crecimiento en la producción. Con liberalización parcial y contingentes para productos del MERCOSUR, los resultados mostraron que estos países no tendrían dificultades para cubrir los contingentes asignados. En el segundo escenario que incluía la liberalización parcial unilateral combinada con la propuesta del G-20 en las negociaciones de la OMC, las exportaciones del MERCOSUR a la UE disminuirían en relación al escenario previo, debido a la competencia con otros países a los cuales también le serían asignadas preferencias. En el tercero, que combinó los dos escenarios anteriores, se dio un fuerte incremento de las importaciones europeas desde el MERCOSUR.

Burrel et al. (2011), en el mismo trabajo citado anteriormente, utilizaron igualmente CAPRI para simular los impactos producidos por los cambios en la política comercial, específicamente en el sector agrícola. La simulación con GLOBE indicó que con la implementación del acuerdo, las ganancias en los sectores no agrícolas serán menores $y$, sus beneficios relativamente pequeños teniendo en cuenta el tamaño de la economía de la UE. El uso de CAPRI, en calidad de modelo equilibrio parcial, permitió simular los impactos que se generarían en la agricultura como consecuencia del acuerdo en ambas partes. Esto es importante por sobre todo teniendo en cuenta que los principales efectos negativos y ajustes en la UE serán en el sector agrícola, especialmente en lo que concierne a los cambios en la redistribución del ingreso entre la agricultura y el resto de la economía, que se vuelve más negativo para la UE al considerar en el modelo que las peticiones del MERCOSUR sean aceptadas por la UE. 
Sus resultados indicaron que las importaciones europeas aumentarian en todas las categorías, excepto en las oleaginosas. Los principales incrementos en el primer escenario (oferta de la UE en septiembre del 2004) correspondió a legumbres $(50,9 \%)$, verduras y frutas $(42,7 \%)$ y aceites vegetales $(33,2 \%)$. En el segundo (exigencias del MERCOSUR del 2006), los principales incrementos se dieron en mantequillas $(336,3 \%)$, carnes, especialmente porcina, $(89,7 \%)$, legumbres $(44,3 \%)$ y frutas (naranjas, melones y banana) y hortalizas, ambas con $43,7 \%$. En relación a los contingentes arancelarios, en ambos escenarios el llenado de cuotas en más del $90 \%$ se dió para todos, excepto trigo, maíz y carne caprina $\mathrm{u}$ ovina, y únicamente en el segundo para carne porcina. En los escenarios que incluyó a Doha los mayores incrementos fueron en las importaciones de carne, porcina y bovina principalmente, que en el último escenario llegó a $139,3 \%$ en relación al año referencial. Las importaciones de mantequilla siguieron el mismo patrón al punto que en el último escenario el incremento con respecto al año de referencias fue del 336,9\%. Igualmente presentaron incrementos los cereales, las frutas y vegetales, así como los aceites. Las semillas y tortas oleaginosas tuvieron valores negativos. En relación a los contingentes arancelarios, en el tercer escenario no llenaron sus cuotas carne de oveja y cabra, en el segundo ocurre lo mismo para trigo, maíz, carne ovina o caprina. En el último escenario, se confirmó el bajo llenado en trigo, maíz y carne ovina o caprina; se dieron leves disminuciones en arroz, carne aviar y mantequilla,y se mantuvo el llenado en carne bovina y en azúcar. Las importaciones de carne porcina quedaron $15 \%$ por debajo de su cuota.

\section{Estudios con indicadores de comercio}

ALADI (2002) combinó el uso de varios indicadores de comercio y dos sistemas de clasificación de mercaderías y Clasificación Uniforme para el Comercio Internacional, con el objetivo de identificar por un lado los productos del comercio regional de Latinoamérica que serían desplazados por las importaciones europeas en el marco de un acuerdo y, aquellos bienes latinoamericanos con oportunidades en el mercado comunitario. Dentro de este último, y para el MERCOSUR, los principales bienes identificados y sus respectivos pesos en el valor total exportado fueron: manufacturas de origen agropecuario (35\%), alimentos $(33 \%)$, y productos químicos y conexos (11\%). La mayor parte de los productos seleccionados para Argentina y Brasil seguían esta tendencia, mientras que los bienes de Paraguay y Uruguay tenían mayor participación de productos agrícolas primarios y menor peso de las manufacturas. Más específicamente, para Argentina se identificaron 65 productos, siendo los de mayor oportunidad: carne equina, miel natural, preparaciones de carne bovina, extractos y jugos de carne, aceites vegetales de maní, olivo, girasol, ricino, maíz, algodón, pescados, frutos cítricos y manzanas. En el caso de Brasil se identificaron 105 productos, siendo los de mayor oportunidad las ceras vegetales, los jugos de frutas, tabaco, café sin tostar, azúcar en bruto, carne, preparaciones de aves y extractos de café, semi-productos de hierro y acero sin alear. Los resultados identificaron 85 productos con oportunidades para el Paraguay: trigo duro, diferentes formas de presentación de carne bovina congelada o refrigerada, maderas aserradas o enchapadas, despojos diversos de carne bovina, tabaco sin desnervar, semillas de maní, cártamo y harina de soja. Finalmente para el caso de Uruguay, la metodología identificó 60 productos con oportunidad: harinas y sémolas de legumbres, sagú y otros tubérculos, lana peinada, carne de especia equina, productos diversos de carne bovina $\mathrm{y}$ ovina, frutos cítricos y arroz.

CEI (2003), estudió el impacto del acuerdo UEMERCOSUR en el comercio exterior de Argentina combinando índices de complementariedad comercial y niveles arancelarios en la UE. El trabajo concluyó que las sub-partidas con mayor oportunidad en el mercado comunitario fueron las que mayor arancel enfrentaron (por encima de 10\%) y fueron bienes agroalimentarios (carnes, frutas y cereales) y de la pesca. Entre las amenazas de parte de la UE en el mercado regional se citaron automóviles, máquinas, lácteos, frutas, harinas y plásticos.

Terra (2001) analizó para Uruguay los obstáculos e identificó los beneficios que el acuerdo traería para ese país. Los indicadores utilizados por la autora fueron ventajas comparativas reveladas e índices de complementariedad comercial. El trabajo concluyó que existía baja coincidencia producto a producto en la oferta exportable del MERCOSUR como bloque. Halló más bien similitud sectorial, con mayor énfasis entre Uruguay y Argentina. Estos fueron productos agropecuarios, alimentos, textiles, curtiembres y materiales de transporte. Por otro lado identificó que las principales importaciones del MERCOSUR correspondieron a bienes de capital y productos de la industria química sujetos a excepciones al AEC y con regímenes. La concreción del acuerdo podría llevar a desvíos del comercio. Finalmente se identificaron 48 productos que Uruguay exportaba al MERCOSUR que 
enfrentarían competencia europea con la firma del acuerdo.

\section{CONSIDERACIONES FINALES}

Los documentos revisados son los principales estudios de impacto del Acuerdo de Asociación Regional (AAR) en su componente comercial; y coinciden en que el MERCOSUR obtendrá ventajas a través del aumento en las exportaciones de los productos agrícolas en los cuales tiene ventaja comparativa muy superior a su contraparte, en este caso la Union Europea. La especialización que propiciarpa el Acuerdo abre la posibilidad de que los países del bloque sudamericano, se conviertan en eternos productores y exportadores de materias primas agrícolas y sus primeras manufacturas.

\section{REFERENCIAS BIBLIOGRAFICAS}

ALADI (Asociación Latinoamericana de Integración, Uruguay). 2002. Probable impacto que tendrían los acuerdos con la Unión Europea en el comercio intrarregional y en el comercio de los países miembros con los países de Europa. Uruguay, ALADI. 183 p. (Estudio 149)

Balassa, B. 1965. Trade liberalization and "revealed" comparative advantages. Manchester School of Economics and Social Studies 33(2):99-123.

Bhagwati, J. 1992. Regionalism versus multilateralism, The World Economy 15(5):535-555.

Boyer, I; Schuschny, A. 2010. Quantitative assessment of a free trade agreement between MERCOSUR and the European Union (en línea). Santiago de Chile, Chile , CEPAL. (Serie Estudios estadísticos y prospectivos $\mathrm{N}^{\circ}$ 69.) Consultado 09 sep. 2013. Disponible en http://www.eclac.org/cgi-

bin/getProd.asp?xml=/publicaciones/xml/1/41551/P415

51.xml\&xsl=/publicaciones/ficha-

i.xsl\&base=/publicaciones/top_publicaciones-i.xsl\#

Burrel, A; et al. 2011. Potential EU-MERCOSUR Free Trade Agreement: Impact Assessment (en línea). Joint Research Centre-EU, Luxembourg Publications Office of the European Union. Consultado 09 set. 2013. Disponible http://ipts.jrc.ec.europa.eu/publications/pub.cfm?id=48 19
CEI (Centro de Economía Internacional, Argentina). 2003. Oportunidades y amenazas para la Argentina de un acuerdo MERCOSUR-Unión Europea: Un estudio de impacto sectorial" (en línea). Buenos Aires, Argentina, CEI. 106 p. (Serie Estudios del CEI. Nro. 3). Consultado 21 ene. 2010. Disponible en http://cei.mrecic.gov.ar/revista/01/revista.pdf

Denzin, NK; Lincoln, YS. 2000. Handbook of qualitative research. 2 ed. Londres, Sage.

Drogue, S; Ramos, M. 2005.Tariff-Rate Quotas and agricultural trade: an application to the agricultural free-trade negotiation between the MERCOSUR and the EU (en línea). Francia, INRA. (Working Papers Economie Publique No 2005/01). Consultado 09 sep. 2013. Disponible en http://EconPapers.repec.org/RePEc:apu:wpaper:2005/0 1

Folgueiras, P; Martínez, M. 2009. El desarrollo de competencias en la Universidad a través del Aprendizaje y Servicio Solidario (en línea). Revista Interamericana de Educación y Democracia 2(1):55-76. Consultado 05 may. 2016. Disponible en http://diposit.ub.edu/dspace/handle/2445/

Kirkpatrick, C; George, C. 2009. Trade sustainability impact assessment (SIA) of the association agreement under Negotiation between the European Community and MERCOSUR (en línea). Manchester, Institute for Development Policy and Management, University of Manchester. Consultado 11 dic. 2009. Disponible en http://www.sia-

trade.org/mercosur/phase2/Mercosur_final_overview_3 10309.pdf

Laborde, D; Ramos, M. 2007. Will regionalism survive multilateralism? The EU-MERCOSUR example (en línea). In Annual Conference on Global Economic Analysis "Assessing the Foundations of Global Economic Analysis". (10, West Lafayette, Estados Unidos de América) West Lafayette, Estados Unidos de América, GTAP, Purdue University. Consultado 09 set. 2013. Disponible en https://www.gtap.agecon.purdue.edu/resources/downlo ad/3355.pdf

Maesso, M. 2011. La integración económica. Revista Boletín Económico de ICE. (Madrid) (858):119-132. Consultado 09 Sep. 2015. 
Monteagudo, J; Watanuki, M. 2003. Regional trade agreements for MERCOSUR: a comparison between the FTAA and the FTA with the European Union. Économie international 3(94-95):53-76. Consultado 21 ene. 2010. Disponible en http://www.cepii.fr/anglaisgraph/publications/economi einter/rev9495/rev9495c.htm

Piermartini, R; Tech, R. 2005. Demystifying modelling methods for trade policy. Ginebra, Suiza, Organización Mundial de Comercio (WTO). 59 p. (Paper $\mathrm{N}^{\circ} 10$ )

Solares, A. 2010. Integración: teoría y procesos: Bolivia y la integración (en línea). La Paz, Bolivia, Eumed. Consultado 9 set. 2015. Disponible en www.eumed.net/libros/2010e/814/

Terra, M 2002. Uruguay y el MERCOSUR frente a un acuerdo con la Unión Europea: prioridades para la negociación arancelaria (en línea). Santiago de Chile, Chile, CEPAL. (Serie estudios e investigaciones) Consultado 9 set. 2015. Disponible en http://www.cepal.org/es/publicaciones/28669-uruguayy-el-mercosur-frente-un-acuerdo-con-la-unioneuropea-prioridades-para-la
Trejos, A. 2009. Instrumentos para evaluación del impacto de acuerdos comerciales internacionales: aplicación para países pequeños en América Latina (en línea). Mexico, Conferencia Económica de las Naciones Unidas para América Latina-Sede México. Consultado 9 set. 2015. Disponible en http://www.eclac.org/cgibin/getProd.asp?xml=/publicaciones/xml/9/37329/P373 29.xml\&xsl=/mexico/tpl/p9f.xsl\&base=/mexico/tpl/top -bottom.xsl

UNCTAD (Conferencia de las Naciones Unidas sobre Comercio y Desarrollo, Suiza). 2012. A practical guide to trade policy analysis. Suiza, Organización Mundial de Comercio. 232 p.

Weissleder, L; Adenäuer, M; Heckelei, T. 2008. Impact assessment of trade liberalization between EU and MERCOSUR countries (en línea). In Seminar modeling of agricultural and rural development policies. (107, Sevilla, Spain). Sevilla, Spain, EAAE. Consultado 9 sep. 2013. Disponible en http://purl.umn.edu/6667 